THREE CASES OF

\section{SUCCESSFUL REMOVAL OF FIBRO-CYSTIC TUMOURS OF THE UTERUS.}

\section{Br THOMAS KEITH, M.D., F.R.C.S.}

Casw 1.-In December, 1873, Miss S-, aged fifty-two, consulted Dr. Perry, of Glasgow, on account of a tumour which she had just discovered in her abdomen. Dr. Perry pronounced it to be a small fibrous tumour of the uterus, and told her that it would probably follow the usual history of such growths at her time of life, and give rise to little or no inconvenience. Two months after this I expressed a similar hope, adding, however, as she dreaded some cancerous affection, that should her health and comfort come to be seriously interfered with, she could look forward to permanent relief by operation. The tumour grew rapidly, and in June, six months after it was noticed, reached above the umbilicus, and was distinctly cystic. Her general health was then wretched, but after two months in the country she improved in strength, and became anxious to have something done before winter, for she had long suffered from bronchitis, and was generally confined to the house during cold weather. Having ohjections to removing small tumours, I put her off from month to morth. She made her appearance in the end of October last, suffering then from cough and bronchial catarrh.

The patient was fair-complexioned, anæmic, extremely emaciated and fragile-looking. She was the youngest of a family of twelve, and had never been strong. At sixteen she had severe pleurisy, on account of which the right side of the chest remained contracted. It was much flattened, and the edges of the ribs were depressed towards the spine, thus interfering to a great degree with the capacity of the abdomen. The tumour touched the edges of the ribs on the right side, and there was now a considerable amount of ascitic fluid. The abdominal wall was very thin; fluctuation was distinct in the right side, where there was a prominent cyst the size of the head. Part of the tumour came .down into Douglas's space; it was there cystic and nonadherent. The cervix uteri was flattened, not enlarged, central, and rather low in the pelvis. Movements of the tumour affected it slightly, just as they affect the cervix in most ovarian tumours surrounded by free fluid. No specially careful examination was made; a single, almost momentary, feeling of the pelvis sufficed, for now I never doubted that the tumour was other than ovarian. It was looked upon as one of those common cases where there had been an escape of fluid from some of the cysts into the cavity of the abdomen, and in consequence chronic peritonitis had been set up. In such cases my practice has for a long time been to advise earlier operation than in cysts not accompanied by free fluid; for experience had taught me that, if of ten tapped, such patients do badly after operation. Besides, I had now an additional reason for early removal of such tumours, for the results of observations made for some time by Dr. Foulis showed that secondary epithelial growths on the peritoneum may originate from escaped epithelial elements from burst cysts, and set up exhaustive peritonitis.*

On the 2nd of November last sulphuric ether was given, the tumour exposed, the ascitic fluid allowed to escape, and the large prominent cyst punctured. Four or five pints of bloody serum slowly escaped. Then by pressure more fluid was got away, the opening enlarged to admit the hand, and the cystic portion in the pelvis somewhat broken up. It was then noticed that the fluid from the cysts coagulated on exposure to the air, and it was evident that the tumour was uterine. The wound was enlarged, and the tumour turned out, along with the left ovary, which was the size of a hen's egg. The uterus spread out rapidly into the cystic mass, the neck being the thickness of the arm, too large to be embraced by any clamp. After freeing the bladder downwards, a steel wire was secured round the lowest part of the neck,

* Edin. Med. Journ., Feb. 1876. just above the vagina, by Koeberlé's instrument. The right ovary was healthy, and was not removed. The tumour was then cut away, and as the stump was pared down as close to the wire as was deemed safe, the cavity of the uterus was opened. It contained a small polypus, and its length was probably less than normal. The wound was closed tightly round the neck, a strong soft needle bent in the middle securing the whole outside. Though the abdominal wall was thin and relaxed, there was tension and depression of the stump towards the sacrum. The growth was a true fibro-cystic tumour of the uterus, and weighed $11 \mathrm{lb}$.

The patient recovered, and returned to Glasgow thirty seven days after operation.

CASE 2.-An unmarried woman, aged forty-four, was admitted in February, 1874, into Ward 16 of the Royal Infirmary, where I saw her at the request of Dr. Matthews Duncan. She was a pale, thin, unhealthy-looking woraan. She had granular everted eyelids, and was half-blind from old inflammations of the cornea. In the neck were cicatrices of suppurated glands. Up till June last her health had been fairly good; she was then obliged to give up her situation as cook in London, where she had lived for more than twenty years. Menstruation had always been regular and normal. Five weeks before admission a tumour bad been detected; it was hard, elastic, quite fixed, and reached to the umbilicus. The cervix uteri was drawn to the left side of the pelvis; it was almost beyond reach of the finger, and felt as if lost in the tumour. No doubt was entertained that the case was one of uterine fibroid. After two months? residence in hospital she was dismissed, all interference being declined.

In the course of the summer she wrote several times to. say that she bad suffered severely, and that the tumour had greatly increased in size. She was unable to do anything for herself, and was anxious to have something tried. Though told that hers was not a case for operation, she came back in the beginning of September. The tumour had increased; it was tender, and had become very prominent on the right side, pushing the loin outwards. The feeling of elasticity was less marked, while that of fluctuation was pretty distinct, and there was some ascitic fluid. The relations in the pelvis were the same, the tumour filling the whole upper pelvis. Notwithstanding the presence of ascitic -fuid, the tumour felt everywhere fixed and immovable. On the 5th of September a puncture was made at the umbilicus; the fluid removed was of a dark-brown colour, depositing on standing a little blood. It contained blood-corpuscles, white bloodcells in large numbers, and some cells resembling pus. Much irritation followed this tapping, and the ascitic fluid increased. After ten days the cyst had become tense, when sixty ounces of the same kind of fluid were withdrawn. The pain was relieved by this, and she was again sent away.

She soon came back, importunate for operation at any risk; her life was miserable from pain, and her health had now given way. The cyst was again tense, and the whole tumour was growing rapidly. It was agreed to make an attempt to remove it, the patient clearly understanding that this might not be accomplished.

On Dec. 12th the tumour was exposed by a free incision. It was of a dusky brown colour, covered by enormous veins. It was firmly attached to the right iliac fossa, right lumbar region, and partly to the abdominal wall. This extent of adhesion quite accounted for the fixed state which the tamour had always presented. The hand could not be passed in to the pelvis below the tumour. Upwards of sixty ounces of dirty reddish serum were removed from the large cyst, withont much diminishing the size. The hand was then passed behind, and with one strong sweep of the arm the whole adhesions were broken up, the incision enlarged, and the tumour turned out of the abdomen, along with the left ovary. In a few minutes she became quite faint, so rapidly was blood lost from the large extent of bleeding surface of the tumour. The pedicle-that is, the neck of the uteruswas treated as in the former case. The broad ligaments were very vascular, but only the left ovary was included in the ligature. From the previous elevation of the cervix. the stump was secured in the lower angle of the wound with less tension than in the previous case. Many bleeding points high up near the posterior margin of the liver, where the adhesions commenced, were secured with much difficulty. The first part of the operation occupied only a few minutes, but it was upwards of two hours ere the wound was clased. 
A glass drainage-tube was left, passing down to the lowest point in the pelvis. The patient was pulseless when placed in bed, and the pulsation did not return in the left radial for upwards of half an hour. This was an anxious operation on account of the unusual loss of blood.

It is unnecessary to give here details of the slow convalescence. The red serum was removed from the pelvis every few hours; the whole amount within the first three days did not exceed three ounces. This the patient might easily have absorbed. During the second and third weeks flatulent distension was great, and there were fears of obstructed intestine. The canal of lymph, which after a few days forms round the drainage-tube, seemed to bave led to the adhesion of folds of intestine at angles, and thus became the occasion of much distress and of no small danger to the patient. As in the previous case, the slough extended beyond the wire, and a very large cavity was left on its separation, which had quite cicatrised in five weeks. Six weeks after operation she went home, and now writes that she is perfectly well in every way.

CASE 3.-This patient was forty years of age, and was sent to me by Dr. Millar, of Londonderry. The case was one of rapidly growing soft cystic fibroid, with pain and profuse hæmorrbages. A very accurate diagnosis was made. The uterus and both ovaries were removed, along with numerous cysts in the broad ligaments. Though quite recovered, the patient has not yet returned to Ireland. An account of this case will be given at some future time.

In the first of these three cases there was a mistake of diagnosis as to the nature of the tumour. It is the only one of 194 operations. Hitherto I had the good fortune to aroid cases of soft fibrous and fibro-cystic tumours of the uterus. But as it has happened to all who have performed ovariotomy many times to come down upon a fibro-cystic tumour of the uterus instead of an ovarian tumour, I knew that I could not always escape; and for many years I have never gone to perform ovariotomy without being at the same time prepared to remove the uterus if necessary. Fortunately in this case the surgical treatment required was the same, for large fibro-cystic tumours of the uterus of ten kill as rapidly as do ovarian tumours.

These are the only cases in which $I$ have interfered with uterine tumours by abdominal section. They are, however, enough to satisf $y$ me that the removal of an enlarged uterus and ovaries is an operation not to be lightly undertaken. Without the knowledge that ovariotomy has given me, the results would probably have been different. Even as it was, in the second case the hæmorrhage was so profuse that the patient was pulseless in a few minutes after the operation was begun. The personal attention afterwards necessary was, in each, greater than in half a dozen average cases of ovariotomy; while the third had such profuse secondary hæmorrhage seven days after operation, that had I not, by mere accident, been on the spot almost immediately after the alarm was given, the patient must have died in a few minutes.

Daring the last twelve or fourteen years, I need hardly say, a large number of uterine tumours of all kinds have passed through my hands; and the advice hitherto given has been to let them alone, either because interference was unnecessary or because their removal was too dangerous. Of course I do not allude to submucous tumours that could be reached by the vagina. I might have removed scores of pediculated fibroids which, surgically speaking, were very tempting; but in these, nothing but almost absolute safety of operation could have justified interference, and they were all declined, though I was often pressed hard to operate. The melancholy results that have hitherto followed the performance of this operation amongst us forbade interference, even when necessary, under the most favourable circumstances, far more in those dangerous cases of large, quickly growing, soft fibroids or fibro-cysts. Though the greater number of uterine tumours-if not malignant-give little trouble, rarely interfering with life, and often not even with the comfort of the patient, yet in a certain number they cause the subjects of them to lead useless, dependent, miserable lives, full of suffering, ending only with life. I hope the time is not far distant when many of these unfortunates will look to surgery for relief with as much confidence as those afflicted with ovarian disease now do.

Edintargh.

\section{HISTORY OF A CASE OF PY AMIA IN} PRIVATE PRACTICE,

IN WHICH RECOVERY TOOK PLACE AFTER EIGHTEEN ABSCESSES HAD OCCURRED.

By FREDERICK H. DALY, M.D.

(Concluded from page 471.)

JUNE 10th to 17th. - The patient's feet became more œdematous and intolerably painful. I tried resting them on an inclined plane, but he said it made them much worse; so I got him one of Salter's swings, with the cradle, and this he found most comfortable. I removed the drainagetubes from the thighs, as there was scarcely any discharge from these wounds. On the evening of the 18th his tem. perature was $102^{\circ}$; pulse 150 . Next evening the temperature was $104^{\circ}$; pulse 160 ; and there was very little discharge from any wound, no sign of a fresh abscess, and nothing to account for the rapid pulse; had no appetite. At 7 A.M. on the 14th he had a prolonged rigor, and there was a deep erysipelatous blush all over the right hip and front of the thigh. His temperature was $105 \cdot 8^{\circ}$. I also discovered a new abscess behind the left trochanter, symmetrical with the one behind the right trochanter. I opened it, and it contained four ounces of unhealthy sanguineous pus. His pulse was feeble, and he looked very badly, almost yellow. The urine was bright-coloured and scanty; no albumen. He had no appetite. There was no discharge from any of the old abscesses.

On the 14th Mr. Hutchinson saw him with me late in the afternoon. The erysipelas had spread all over the back, and to the left side and thigh. Mr. Hutchinson and Mr. Waren Tay examined his blood microscopically, and there was a relatively large number of white corpuscles. In consultation, we agreed to stop the mercury, which he had taken for exactly a month without its producing any effect upon the gums or breath, and again to give him twenty grains of quinine daily with the half-ounce of tincture of steel, and to press flaid nourishment and stimulants. Strong lead-and-spirit lotion to be continually applied to the erysipelatous parts. The erysipelas lasted until the 21 st of June, and during this period it continued travelling about from part to part. His prostration was extreme. He became quite deaf, and ultimately became so exhausted that he could scarcely bear the application of the lotion. The nervous prostration and muscular exhaustion were very great, his hands and tongue were tremulous, and the hyperæsthesia was exquisite. Mr. Hutchinson saw him with meduring this period, and we despaired of his life, but the free use of stimulants kept him alive. During each twenty-four hours he took twenty-four ounces of port wine, a pint of champagne, and eight ounces of brandy, together with milk, beef-tea, and beaten up-eggs. Some days he was almost in a state of collapse. His urine kept free from albumen, and his temperature was never below $103^{\circ}$, or his pulse below 140 .

On the 20th the erysipelas was quite gone, and the same day the left thigh discharged about a drachm of pus, all discharge from the abscesses having ceased during the attack of erysipelas.

On the 21st, his morning temperature was down to $101^{\circ}$; pulse 132. Mr. Hutthinson, when he saw him with me in the afternoon, expressed the most unfavourable opinion of the case. We found him deaf, sleepy, and dull, dozing to sleep while you were speaking to him ; no albumen in urine, sp. gr. 1022 ; feet and legs greatly swollen; his voice was altered, and he looked almost cadaverous; heart and lung sounds normal. Mr. Hutchinson detected fluid in the right knee-joint, and the knee-cap grated, but he did not think the fluid was pus, probably only discoloured flaky synovial fluid. The right knee measured three-quarters of an inch more than the left. We increased his port wine to three pints during twenty-four hours, in addition to the brandy and champagne. The extra amount of stimulant agreed with him. Next day he had rallied a little; he was not so sleepy or deaf, and his tongue was cleaner. He took his 